\title{
Abordaje epistemológico en la investigación social para la producción de teorías científicas
}

\section{Epistemological approach in social research for the production of scientific theories}

Recibido: 04/11/2020

Aceptado: 02/06/2021

Publicado: 30/06/2021
Luis Gerardo Doubront dr.doubront@gmail.com

https://orcid.org/0000-0003-4174-2169

Universidad Nacional Experimental Simón Rodríguez (Venezuela)

Marcel Alejandro Doubront marceldoubront2@gmail.com https://orcid.org/0000-0002-6764-2708

Universidad Nacional Experimental Simón Rodríguez (Venezuela)

Andrés Elias Gómez Alfonzo andreselias18@gmail.com

https://orcid.org/0000-0002-6831-9021 Investigador independiente (Venezuela)

Resumen: El estudio tiene el propósito de dilucidar el abordaje epistemológico en la investigación social para la producción de teorías científicas, tomando en cuenta las debilidades conceptuales en la formación de investigadores que recurrentemente se reducen entre un abordaje cuantitativo o cualitativo como eje orientador del proceso investigativo. La metodología empleada se enmarcó en un enfoque cualitativo, utilizando el método hermenéuticocritico que permitió interpretar la información en unidades de análisis a través 
de fuentes documentales. En este sentido, los hallazgos de la investigación reseñaron la importancia de conocer la percepción del investigador frente a la realidad social, la relación entre el investigador y lo investigado para acceder al conocimiento científico y la estrategia procedimental del investigador para responder los objetivos del estudio. En conclusión, los enfoques metodológicos cuantitativos o cualitativos son el resultado derivado de todo un proceso ontológico, epistemológico y metodológico que orienta la investigación, adquiriendo relevancia solo procedimentalmente.

Palabras clave: Abordaje epistemológico, Producción de teorías científicas, Ciencias sociales, Evaluación de teorías, Investigación social.

Abstract: The study aims to elucidate the epistemological approach in social research for the production of scientific theories, taking into account the conceptual weaknesses in the training of researchers that recurrently are reduced between a quantitative or qualitative approach as the guiding axis of the investigative process. The methodology used was framed in a qualitative approach, using the hermeneutical-critical method that allowed interpreting the information in units of analysis through documentary sources. In this sense, the research findings highlighted the importance of knowing the researcher's perception of social reality, the relationship between the researcher and what is being investigated to access scientific knowledge and the investigator's procedural strategy to respond to the objectives of the study. In conclusion, quantitative or qualitative methodological approaches are the result derived from an entire ontological, epistemological and methodological process that guides the research, acquiring relevance only procedurally.

Key words: Epistemological approach, Production of scientific Theories, Social sciences, Theory evaluation, Social investigation.

\section{Introducción}

Hablar de abordaje epistemológico en la investigación social se convierte en una necesidad en la naturaleza de las ciencias sociales, debido a que muchos autores aseguran que la producción de teorías científicas obedece a criterios de verdad circunscritos en la filosofía de la ciencia. Según Paz-Sandín (2003), 
"una perspectiva epistemológica es una forma de comprender y explicar cómo conocemos lo que sabemos: ¿Qué tipo de conocimiento obtendremos en una investigación? ¿Qué características tendrá ese conocimiento? ¿Qué valor puede otorgarse a los resultados obtenidos?” (p.47). Por su parte, Crotty (1998) identifica tres orientaciones epistemológicas fundamentales circunscritas en los paradigmas o perspectivas teóricas de la investigación: “el objetivismo, el construccionismo y el subjetivismo" (p.7).

Sin embargo, la formación de investigadores presenta un cúmulo de dificultades conceptuales para la producción de teorías científicas, siendo recurrente escuchar en los pasillos de las universidades hablar de paradigma cuantitativo o cualitativo, sin considerar el abordaje epistemológico como fuente del conocimiento científico para la producción de teorías. Según Hughes (1980, como se citó en Corbetta, 2007), "cada procedimiento o instrumento de investigación está entrelazado de manera inextricable con las interpretaciones concretas del mundo que tiene el investigador y su modo de conocer ese mundo" (p.13). Así pues, esta consideración requiere de una reflexión científica donde se posicione al investigador, convirtiéndose en un ente indisoluble la metodología de la epistemología.

La discusión de fondo entre métodos cuantitativos y cualitativos no es metodológica. Solo tiene sentido planteada epistemológicamente y, únicamente desde la epistemología, puede ser resuelta. Y, desde esta, se advierte que la cuestión no está ni en lo cualitativo ni tampoco en lo cuantitativo, sino en cómo se relacionan ambas vertientes de lo real. Justamente esta relación se encuentra hoy en un proceso de cambio (Munné, 1995, como se citó en Paz-Sandín, 2003).

Según lo expuesto, la orientación del proceso investigativo se fundamenta en el abordaje epistemológico. Sin embargo, existen autores que contradicen esta posición. "Las divisiones epistemológicas y de paradigmas no contribuyen en nada a la ciencia (salvo que se utilicen con fines clasificatorios o taxonómicos), ya que la complejidad de los problemas que aquejan a la sociedad humana son muchos" (Ortiz, 2013, p.18).

En el modelo que sigue esta investigación, la distinción entre investigación cualitativa e investigación cuantitativa sucede en el nivel de métodos. Lo que ocurre en otros niveles es una diferenciación entre la investigación objetivista/positivista, por un lado, y la investigación construccionista o subjetivista, 
por el otro. Sin embargo, en la mayoría de libros de texto, son la investigación cualitativa y la investigación cuantitativa las que se enfrentan entre sí como polos opuestos (Crotty, 1998).

En este escenario, la formación de investigadores para la producción de teorías científicas, en las ciencias sociales, se ha envuelto en una nebulosa de conceptos que requieren clarificarse. Foucault (1979) establece que un investigador debe ofrecer un análisis lógico en el modo de proceder. "Analizar positividades, es mostrar de acuerdo con qué reglas una práctica discursiva puede formar grupos de objetos, conjuntos de enunciaciones, juegos de conceptos, series de elecciones teóricas" (p.305). El citado autor marca sus herramientas con su signo especial: reflexión sobre la producción de conocimientos científicos. En ese sentido, se puede hablar de una epistemología que, en tanto proceso para la producción de conocimientos, no le rinde cuentas al cientificismo. La epistemología es vía obligada para hacer teoría (Perdomo, 2009).

En atención a lo expuesto, la problemática se reduce a la necesidad de cuestionar y clarificar elementos teóricos conceptuales para abordar la investigación social, tomando en cuenta la necesidad de incorporar los símbolos significantes del lenguaje en la línea discursiva del investigador para la acción comunicativa. Es por ello la necesidad de dilucidar cómo se manifiesta el abordaje epistemológico en la investigación social para la producción de teorías científicas. Esto permite interpretar la percepción de la realidad por parte del investigador, la relación entre el investigador y lo investigado, al igual que la estrategia procedimental para fundamentar la legitimidad de los axiomas para la producción de teorías científicas en la investigación social.

\section{Metodología}

Esta investigación se desarrolló a través de un abordaje epistemológico racional-idealista, como eje orientador del proceso investigativo. Se utilizó el método hermenéutico crítico como eje procedimental, con la intención de interpretar los símbolos significantes del lenguaje para la acción comunicativa. Según Doubront \& Doubront (2020), "el marco del método de investigación hermenéutico, proveniente del griego 'hermeneutiqué', que significa en latín 'interpretâri', referenciando al arte de interpretar los textos”' (p.4). 
Para los hermenéuticos críticos, la representación del significado de una expresión es relativamente no problemática. Lo importante es la valoración de los significados de dicha expresión a la luz de condiciones históricas. La investigación se ocupa de clarificar las condiciones bajo las que una comprensión distorsionada puede haber ocurrido; una clarificación que debe conducir a una acción práctica emancipadora. En tal sentido, corresponden los trabajos de Habermas como paradigmas de esta postura (Paz-Sandín, (2003).

En cuanto al análisis cualitativo, la investigación documental no solo es una técnica de recolección y validación de información, sino que además constituye una de sus estrategias, la cual cuenta con particularidades propias en el diseño del proyecto, la obtención de la información, el análisis y la interpretación. Como estrategia cualitativa, combina diversas fuentes (primarias y secundarias) (Galeano, 2012). En este sentido, en esta investigación se recolectó la información aplicando la técnica de revisión sistemática, con la cual se consultaron a diversos autores a través de una muestra teórica; esto con el objetivo de extraer información inherente a responder una pregunta de investigación desde una posición investigativa, teórica y filosófica, dando lugar a la técnica de triangulación teórica para el análisis de la sistematización en un proceso de categorización, estructuración, contrastación y hermeneusis (teorización).

\section{Resultados}

Se toma como referencia lo expuesto por Guba \& Lincoln (1998), quienes teorizan que "los paradigmas de investigación definen para los investigadores qué es lo que están haciendo y qué cae dentro y fuera de los límites de una investigación legítima" (p.5). Por otra parte, para Dendaluce (1999, citado en Paz-Sandín, 2003), la dimensión epistemológica no es considerada por los investigadores de manera frecuente, pese a que se halla en la base de un nutrido número de comportamientos y decisiones de corte metodológico.

En atención a las referencias expuestas, el abordaje epistemológico en la investigación social no puede interpretarse como un ente aislado de lo que se pretende investigar. Esto debido a que adoptar una posición epistemológica registra consecuencias en el abordaje investigativo, que impacta en la percepción del investigador frente a la realidad social, la relación entre el investi- 
gador y lo investigado y la estrategia metodológica del investigador. En tal sentido, se fundamenta la elaboración de las unidades de análisis:

\section{Tabla 1}

Cuadro 1: unidades de análisis

\begin{tabular}{|c|c|}
\hline Percepción del investigador & Plano ontológico \\
\hline Relación del investigador & Plano epistemológico \\
\hline Metodología del investigador & Plano metodológico \\
\hline
\end{tabular}

Nota: Elaboración propia.

\section{Tabla 2}

Matriz de sistematización para la triangulación teórica: percepción del investigador frente a la realidad social

\begin{tabular}{|c|c|c|}
\hline Cuadro 2 & $\begin{array}{r}\text { ¿Cuál es la perce } \\
\text { frente a la }\end{array}$ & dad social? \\
\hline estigativa & Posición teórica & Posición filosófica \\
\hline $\begin{array}{l}\text { Fuente 1: las convicciones acerca } \\
\text { de las relaciones del sujeto-in- } \\
\text { vestigador con la realidad objeto } \\
\text { observable se concretan igual- } \\
\text { mente en dos valores, como son, } \\
\text { el "idealismo" y el "realismo" } \\
\text { (De Berrios \& Briceño, 2008). } \\
\text { Fuente 2: responder a las pre- } \\
\text { guntas ¿qué es la realidad? y } \\
\text { ¿qué es la representación?, con- } \\
\text { lleva a un asunto eminentemen- } \\
\text { te ontológico. Existen, al menos, } \\
\text { dos corrientes de interpretación } \\
\text { de la realidad: una que es de } \\
\text { tipo positivista, es decir natural, } \\
\text { materialista u objetivista; y otra } \\
\text { que es de tipo subjetiva, social, } \\
\text { constructivista o intersubjetivis- } \\
\text { ta (Hincapié, (2017). } \\
\text { Fuente 3: la que es la primera } \\
\text { hipótesis de carácter ontológi- } \\
\text { co hace referencia directa a la } \\
\text { interpretación de la realidad } \\
\text { o de la verdad en la }\end{array}$ & $\begin{array}{l}\text { Fuente 6: la posición del posi- } \\
\text { tivismo de realismo ingenuo, al } \\
\text { suponer en sus principios una } \\
\text { realidad externa objetiva sobre } \\
\text { la cual pueden converger las in- } \\
\text { vestigaciones; al realismo críti- } \\
\text { co del postpositivismo, que aún } \\
\text { supone una realidad objetiva, } \\
\text { pero que acepta que solo se le } \\
\text { puede comprender imperfecta } \\
\text { y probabilísticamente. De igual } \\
\text { manera, al realismo histórico } \\
\text { de la teoría crítica, que supone } \\
\text { una realidad comprensible que, } \\
\text { en términos generales, consiste } \\
\text { en estructuras históricamente } \\
\text { situadas que son, en la ausen- } \\
\text { cia del "insight", tan limitantes } \\
\text { como si fueran reales. Se suma } \\
\text { también al relativismo del } \\
\text { constructivismo, que supone } \\
\text { realidades sociales múltiples, } \\
\text { comprensibles y, en ocasiones, } \\
\text { opuestas, que son producto del } \\
\text { intelecto humano, pero que } \\
\text { pueden cambiar al volverse sus }\end{array}$ & $\begin{array}{l}\text { Fuente 8: al determinar al su- } \\
\text { jeto, el objeto se muestra inde- } \\
\text { pendiente de él, trascendente a } \\
\text { él. Aquí cabe decir que todo co- } \\
\text { nocimiento menta ("intende") } \\
\text { un objeto, que es independiente } \\
\text { de la conciencia cognoscente. } \\
\text { El carácter de trascendentes es } \\
\text { propio, por ende, a todos los ob- } \\
\text { jetos del conocimiento. Se divi- } \\
\text { den los objetos en reales e idea- } \\
\text { les. Se llama real a todo lo que es } \\
\text { dado en la experiencia externa } \\
\text { o interna o se infiere de ella. Los } \\
\text { objetos ideales se presentan, } \\
\text { por el contrario, como irreales, } \\
\text { como si fueran meramente pen- } \\
\text { sados (Hessen, 1999). } \\
\text { Fuente 9: las concepciones } \\
\text { filosóficas de la antigüedad } \\
\text { grecorromana, tanto Platón } \\
\text { como Aristóteles, y del me- } \\
\text { dievo cristiano, son realis- } \\
\text { tas; por ende, dan por sen- } \\
\text { tado que existe una realidad }\end{array}$ \\
\hline
\end{tabular}


sociología en sus aspectos filosóficos, de la siguiente forma: la aparición de la corriente sociológica del interaccionismo simbólico a finales de la década de los años 60, ha propiciado un cambio metodológico en las ciencias sociales y humanas sobre cómo se entiende la realidad y la verdad, de tal forma que la comprensión inicial de una realidad objetiva, se ha desplazado hacia una comprensión de una realidad interpretada y construida subjetivamente (Vidal, 2013).

Fuente 4: el primer punto de vista constituye, en esencia, una interpretación idealista del conocimiento científico, asociada a una base de subjetividad; mientras que el segundo, una interpretación realista del conocimiento científico, está asociado a una base de objetividad. Evidentemente, entre ambas posiciones existen una serie de matices intermedios que van desde el idealismo "absoluto o dogmático", hasta el "realismo ingenuo" y el "realismo crítico" (Camacho, Fortaines \& Urdaneta, 2005). constructores más informados y sofisticados (Guba \& Lincoln, 1998).

Fuente 7: el realismo (una ontológica noción que afirma que las realidades existen fuera de la mente) a menudo se toma implicado al objetivismo (una noción epistemológica que afirma que el significado existe en los objetos, de manera independiente de cualquier conciencia). En algunos casos incluso se encuentra el realismo identificado con el objetivismo. Guba \& Lincoln ciertamente postulan un vínculo necesario entre los dos, cuando afirman que si, por ejemplo, se asume una realidad "real", la postura del conocedor debe ser una de desapego objetivo o libertad de valores para ser capaz de descubrir "cómo son realmente las cosas" y "cómo funcionan realmente las cosas" (Crotty, 1998). fuera de la mente. En tal sentido, el filósofo Aristóteles llamaba a la realidad como substancia; mientras que Platón la denominaba idea. Por otra parte, Descartes y Hume son realistas en este sentido, puesto que creen que la ciencia no hace sino reflejar lo real exterior a la mente (Severini, 1961).

Fuente 10: todas las posturas idealistas son modernas. Algunas corrientes idealistas: Berkeley sostenía que no había realidad alguna fuera de la mente, pero no de nuestra mente, sino de la mente de dios. Por su lado, Kant sostenía que lo real que llamamos el objeto era el producto de una síntesis entre las formas puras del sujeto y el contenido dado en la experiencia. El idealismo, en líneas simplificadas, sostiene que el sujeto determina al objeto en la relación de conocimiento; solo podemos conocer aquello que se amolda a nuestras formas subjetivas de conocer. Esta postura se llama idealista, porque sostiene que es el pensamiento o idea lo que constituye a las cosas como objetos de conocimiento (Chaves \& Gadea, 2018).

Nueva perspectiva: los resultados de la investigación expresan que la percepción del investigador, frente a la realidad social, es inherente a la connotación ontológica del ser expresada entre el "idealismo y el realismo", asumiendo que el idealismo es una corriente filosófica con diversos matices que se traduce en la posición del ser como resultado de su pensar para percibir la realidad tomando un carácter subjetivo. El realismo, mientras tanto, es otra corriente filosófica con diversos matices que condiciona el pensar previo a la posición del ser para percibir la realidad objetivamente.

En síntesis, el investigador, en su abordaje, pudiera adoptar dos perspectivas incompatibles de la realidad: Idealismo/Subjetivista versus Realismo/Objetivista.

Nota: Elaboración propia. 


\section{Tabla 3}

\section{Matriz de sistematización para la triangulación teórica: relación entre el in- vestigador y lo investigado}

\begin{tabular}{|c|c|c|}
\hline Cuadro 3 & \multicolumn{2}{|c|}{ ¿Cuál es la relación entre el investigador y lo investigado? } \\
\hline Posición investigativa & Posición teórica & Posición filosófica \\
\hline $\begin{array}{l}\text { Fuente 1: si los sujetos cog- } \\
\text { noscentes solo contamos con } \\
\text { nuestra razón y nuestros senti- } \\
\text { dos para relacionarnos con los } \\
\text { objetos por conocer en todas y } \\
\text { cada una de las posibilidades de } \\
\text { realización del espíritu humano } \\
\text { (ciencia, arte, religión y filoso- } \\
\text { fía), solo tenemos dos posibili- } \\
\text { dades para establecer contacto } \\
\text { con la cuestión sobre la relación } \\
\text { de conocimiento entre el sujeto } \\
\text { cognoscente y el objeto por co- } \\
\text { nocer: la razón y los sentidos } \\
\text { (Nava, (2017). } \\
\text { Fuente 2: el encuentro de las } \\
\text { distinciones conocidos como } \\
\text { "gnoseológicas" y, por otro lado, } \\
\text { las "ontológicas" conduce a } \\
\text { cuatro enfoques epistemológi- } \\
\text { cos, como campos epistémicos } \\
\text { desde donde se pueda orientar } \\
\text { la investigación científica. Se } \\
\text { observa dentro de estos campos } \\
\text { epistémicos, los denominados } \\
\text { enfoques epistemológicos que } \\
\text { dan cuerpo al plan de trabajo } \\
\text { tanto para la apropiación del co- } \\
\text { nocimiento o dominio de la teo- } \\
\text { ría, como para la apropiación de } \\
\text { la información o dominio de la } \\
\text { práctica (De Berrios \& Briseño, } \\
\text { 2008). } \\
\text { Fuente 3: se describen y comen- } \\
\text { tan elementos que caracterizan } \\
\text { a los estilos de pensamiento de } \\
\text { las personas (Inductivo Con- } \\
\text { creto, Deductivo Abstracto, In- } \\
\text { tuitivo Vivencial, Intuitivo no } \\
\text { Vivencial) y cómo estos se rela- }\end{array}$ & $\begin{array}{l}\text { Fuente 4: en el empirismo hay } \\
\text { una familia formada por los } \\
\text { enfoques Vivencialista-Expe- } \\
\text { riencialista (como el de la et- } \\
\text { nografía, entre otros) y Empi- } \\
\text { rista-Inductivista (como el del } \\
\text { positivismo), ya que tienen en } \\
\text { común su énfasis en la realidad } \\
\text { inmediata que rodea al inves- } \\
\text { tigador y que es captada por } \\
\text { los sentidos. Ambos enfoques } \\
\text { basan el inicio de todo conoci- } \\
\text { miento en los hechos fácticos, } \\
\text { en los datos primarios. En cam- } \\
\text { bio, en el racionalismo hay una } \\
\text { cierta afinidad entre los enfo- } \\
\text { ques Vivencialista-Interpretati- } \\
\text { vista y Racionalista-Deductivis- } \\
\text { ta, en el sentido de que ambos } \\
\text { basan el inicio del conocimiento } \\
\text { en los contenidos mentales (no } \\
\text { en los datos primarios de la } \\
\text { experiencia) y, además, suelen } \\
\text { confiar mucho más en la inves- } \\
\text { tigación basada en el trabajo } \\
\text { mental (interpretación, en un } \\
\text { caso, y deducción, en el otro) } \\
\text { que en los llamados "trabajos de } \\
\text { campo". Se puede hablar solo de } \\
\text { dos tipos: el enfoque basado en } \\
\text { datos de la experiencia y el enfo- } \\
\text { que basado en procesos menta- } \\
\text { les (Padrón, 2014). } \\
\text { Fuente 5: se afirma que cada } \\
\text { postura epistemológica es un } \\
\text { intento de explicar cómo obte- } \\
\text { nemos un determinado cono- } \\
\text { cimiento de la realidad y de de- } \\
\text { terminar el estatus que se debe } \\
\text { asignar a las interpretaciones } \\
\text { que realizamos y las compren- }\end{array}$ & $\begin{array}{l}\text { Fuente 6: ¿cómo se establece la } \\
\text { relación de conocimiento en- } \\
\text { tre el sujeto cognoscente y el } \\
\text { objeto por conocer? Muchos } \\
\text { filósofos se han formulado esta } \\
\text { pregunta y la han resuelto en } \\
\text { diferentes sentidos: para algu- } \\
\text { nos pensadores, por ejemplo, el } \\
\text { sujeto cognoscente determina al } \\
\text { objeto por conocer (Descartes, } \\
\text { 1981; Leibniz, 1991). Otros, por } \\
\text { su lado, dicen que es el sujeto el } \\
\text { que determina al objeto (Locke, } \\
\text { 1994; Hume, 1992). Un conside- } \\
\text { rable porcentaje de pensadores } \\
\text { opinan que ambos elementos } \\
\text { del conocimiento se determi- } \\
\text { nan de manera recíproca, con la } \\
\text { salvedad de que primero se da } \\
\text { el indeterminismo (Aristóteles, } \\
\text { 1992; Santo Tomás de Aquino, } \\
\text { 1991) o que primero aparece } \\
\text { el determinismo (Kant, 1996; } \\
\text { Nava, 2017). }\end{array}$ \\
\hline
\end{tabular}


cionan con los enfoques epistemológicos que muestran los científicos. En ellos, al menos uno de los tres primeros estilos de pensamiento se vuelve predominante, dando lugar de manera correlativa a tres enfoques epistemológicos: el Empirista Inductivo, el Racionalista Deductivo, Introspectivo Vivencial, racionalista-subjetivista (Yánez, 2018). siones que alcanzamos, (Crotty, 1998) identifica tres perspectivas epistemológicas: el objetivismo, por un lado; el construccionismo; por el otro, y, finalmente, el subjetivismo ( $\mathrm{Paz}$ Sandín, (2003). procedían las más veces de la matemática, una ciencia ideal; los empiristas, por el contrario, de las ciencias naturales, ciencias reales. Unos y otros tendrían también completa razón si, acaso, limitasen la postulación de sus teorías epistemológicas a aquella esfera del conocimiento que tienen a la vista (Hessen, 1999).

Nueva perspectiva: los resultados de la investigación expresan que la relación entre el investigador y lo investigado para acceder al conocimiento científico, es inherente a la postura epistemológica adoptada en el proceso investigativo, ya que se manifiestan diversas formas de abordaje para conocer la realidad en estudio, tomando en cuenta que la vía para acceder al conocimiento científico se expresa a través de procesos mentales (racionalismo) o a través de la experiencia sensible (empirismo). En consecuencia, se origina una relación entre la posición del investigador y la fuente del saber (Ser/ Saber; Ontología/gnoseología), originando una relación Investigador/Investigado en cuatro formas predominantes:

- Empírico/Inductivo, Empírico/Analítico o Empírico/Realista, atendiendo al Positivismo/Objetivismo.

- Racionalista/Deductivo, Racionalista/Crítico o Racionalista/Realista, atendiendo al Postpositivimo/Objetivismo.

- Introspectivo/Vivencial, Vivencialista/Experiencialista o Empírico/Idealista, atendiendo al Interpretativismo/Construccionismo.

- Racional/Subjetivista, Racional/Dialéctico o Racional/Idealista, atendiendo al Criticismo/Subjetivista.

Nota: Elaboración propia.

\section{Tabla 4}

Matriz de sistematización para la triangulación teórica: definición de la metodología del investigador

\begin{tabular}{|c|c|c|}
\hline Cuadro 4 & \multicolumn{2}{|c|}{ ¿Cómo se define la metodología del investigador? } \\
\hline Posición investigativa & Posición teórica & Posición filosófica \\
\hline $\begin{array}{l}\text { Fuente } 1 \text { : cualquier investiga- } \\
\text { ción (cuantitativa o cualitativa) } \\
\text { requiere de rigor científico y } \\
\text { ético. La rigurosidad no tiene } \\
\text { relación con la rigidez, pues- } \\
\text { to que el rigor se relaciona con } \\
\text { el respeto por los elementos }\end{array}$ & $\begin{array}{l}\text { Fuente } 4 \text { : la metodología dis- } \\
\text { cute los fundamentos episte- } \\
\text { mológicos del conocimiento, el } \\
\text { papel de los valores, la idea de } \\
\text { causalidad, el papel de la teoría } \\
\text { y su vinculación con lo empí- } \\
\text { rico, la definición y validez o }\end{array}$ & $\begin{array}{l}\text { Fuente 6: a pesar de lo extenso } \\
\text { del debate y de las diversas acep- } \\
\text { ciones que giran en torno a la } \\
\text { dicotimización "para acercarse } \\
\text { a la realidad social", es posible } \\
\text { centrarse en la distinción más } \\
\text { generalizada en la sociología y }\end{array}$ \\
\hline
\end{tabular}


básicos de la investigación, donde se debe tener en cuenta el rigor desde lo epistémico, lo metodológico y lo teórico. El investigador debe ser consciente de su forma de ver el mundo y sus limitaciones, cumplir con las condiciones que exige el método y de la rigurosidad teórica de la cual surgen las preguntas esenciales para enunciar ( $\mathrm{Ra}$ mírez \& Zwerg, (2012).

Fuente 2: asimismo, es necesario considerar la relación contingente entre las maneras de recopilar la información y las preguntas de investigación. Esto genera un sentido coherente y lógico de la metódica asumida en el proceso investigativo y debe complementarse, de manera congruente, con los demás aspectos del diseño propuesto. También, hay que subrayar el sentido epistemológico y ontológico de las técnicas e instrumento de recolección de información, de manera que se facilite la comunicación socializada en virtud de los hechos que se investigan, (Meléndez \& Pérez, 2006).

Fuente 3: se defiende la idea de que las dimensiones deben ser vistas como escogencia relativamente independiente en el proceso de investigación; y que la escogencia cuantitativa o cualitativa es primariamente una decisión sobre la generación de datos y los métodos de análisis, solo secundariamente una escogencia sobre el delineamiento de la investigación o de intereses del conocimiento (Bauer \& Gaskell, 2008). aceptabilidad del recorte de la realidad, el uso y el papel que juegan la deducción e inducción, cuestiones de verificación y falsación, y los contenidos y alcances de la explicación e interpretación. No menos importante, también trata cuestiones como el papel del investigador, en general sus orientaciones culturales y especialmente sus valores, su interacción con los agentes sociales, y las diferencias y superposiciones entre los niveles macro y microsociales (Sautu, 2005).

Fuente 5: existe un acuerdo generalizado sobre la existencia de dos grandes marcos de referencia globales que han orientado la investigación social desde su nacimiento: la perspectiva empirista y la humanista (las etiquetas son muy variadas, destacando entre ellas el objetivismo y subjetivismo; aquí se utilizará el término canónico de positivismo $y$, aunque esté menos consolidado, el de interpretativismo). Se trata de dos divisiones orgánicas y claramente opuestas a la realidad social y los modos de conocerla, que han generado dos conjuntos de técnicas de investigación coherentes y muy diferenciados entre sí. Antes de describirlas, se deben conocer sus orígenes filosóficos para, de forma correcta y clara, poder comprenderlas correctamente (Corbetta, 2007). que se extiende a otras ciencias sociales y de la educación. La noción 'durkheniana' de investigación cuantitativa, fundada en la noción objetivista de los hechos sociales a partir de lógicas explicativas; y la weberiana, a través de la cual se otorga importancia a la interpretación y comprensión de la acción social, proporcionando un papel protagónico a la subjetividad (Cárcamo, Méndez \& Rebolledo, 2008).

Fuente 7: lo que se pide aquí no es solo una descripción de la metodología, sino también una explicación del fundamento que proporciona para la elección de métodos y las formas particulares en las que se emplean los métodos. Llevar o realizar una investigación etnográfica, por dar un ejemplo. La investigación etnográfica, en el espíritu del interaccionismo simbólico, busca descubrir significados y percepciones sobre la parte de las personas que participan en la investigación, viendo estos entendimientos en el contexto de la cosmovisión general de la gente o 'cultura'. De acuerdo con este enfoque, el investigador se esfuerza por ver cosas desde la perspectiva de los participantes. Es esto lo que hace sentido de la intención declarada del investigador de realizar entrevistas no estructuradas y de utilizar una forma no directiva de cuestionamiento dentro de ellas (Crotty, (1998). 
Nueva perspectiva: los resultados de la investigación expresan que se define la metodología del investigador orientado por la realidad social que se pretende abordar y la fuente del conocimiento al que se pretende acceder. Es decir, corresponde a una orientación (empirista o racionalista) en el abordaje como fuente del conocimiento (subjetivista u objetivista), en la percepción de la realidad social (cuantitativo o cualitativo), en la recolección de los datos de la información (explicativa o interpretativa) y en el procedimiento del investigador para valorar los resultados.

En síntesis, el enfoque metodológico de la investigación, cuantitativo o cualitativo, representa la consecuencia de todo un proceso coherente que enuncia el rigor del investigador en la percepción ontológica, relación epistemológica y definición metodológica circunscrita en cada paradigma investigativo.

Nota: Elaboración propia.

\section{Hermeneusis de la investigación}

Los hallazgos de la investigación exponen que el abordaje epistemológico mantiene una relación intrínseca con el sujeto y su forma de percibir la realidad para adaptar una estrategia metodológica que le permita alcanzar los objetivos de la investigación. En tal sentido, existen diversas reglas para acceder al conocimiento científico orientado por el abordaje epistemológico, que contemplan los axiomas para la producción de teorías científicas en las ciencias sociales a través de un modelo, aproximación, constructo o perspectiva teórica. Esto tomando en cuenta la necesidad de incorporar la matriz epistémica discursiva que devela la forma en que el investigador pudo acceder al conocimiento científico y legitimar sus postulados a través de una coherencia ontológica, epistemológica y metodológica que conlleva a una verdad teórica.

Según Guba \& Lincoln (1998), “ahora parece que ha quedado establecido más allá de cualquier objeción que las teorías y los hechos son bastante interdependientes, es decir, que los hechos solo lo son dentro de algún marco teórico" (p.6). En atención a lo expuesto, la producción de teorías científicas se puede llevar a cabo desde cualquier paradigma de la investigación social, tomando en cuenta los siguientes supuestos:

- A nivel ontológico, el abordaje en la investigación social para la producción de teorías debe expresar la percepción del investigador (el ser) frente al fenómeno u objeto de estudio, asumiendo una concepción "subjetivista u objetivista" para conocer la naturaleza del fenómeno a investigar. En este sentido, los hallazgos de la investigación reflejan que la forma y naturaleza en que se puede conocer la realidad es a través del Idealismo/Subjetivista o desde la perspectiva del Realismo/Objetivista. 
- A nivel epistemológico, el abordaje en la investigación social para la aproximación, constructo, modelo o perspectiva teórica debe saber expresar la relación entre el investigador y lo investigado (el ser y el saber), tomando en cuenta la dimensión ontológica (Idealismo/Subjetivista) y la del Realismo/ Objetivista, combinadas con las fuentes del saber para acceder al conocimiento (racionalismo) y (empirismo). En este sentido, la relación se expresa a través del Empirico/Inductivo, Empírico/Analítico o Empírico/Realista; también del Racionalista/Deductivo, Racionalista/Crítico o Racionalista/Realista; del Introspectivo/Vivencial, Vivencialista/Experiencialista o Empírico/Idealista; $\mathrm{y}$, finalmente, de la dimensión Racional/Subjetivista, Racional/Dialéctico o Racional/Idealista.

\subsection{Modelo teórico en la investigación social}

El modelo teórico en la investigación social se desarrolla a través del abordaje epistemológico (Empirico/Inductivo, Empírico/Analítico o Empírico Realista), circunscrito en el paradigma positivista. En el abordaje de la investigación social, se define el término "modelo teórico", ya que concibe el conocimiento científico como una exacta reproducción inmutable de la realidad que puede ser replicada en cualquier tiempo y espacio. En este sentido, el investigador asume una posición objetiva de la realidad donde la aprehensión del conocimiento se expresa fuera de los valores y prejuicios que posea el investigador, siendo validados por la experiencia sensible al comprobar la hipótesis a través de la verificación empírica en una relación dualista sujeto-objeto.

La connotación ontológica (Ser) adopta una posición realista, mejor conocida como "realismo ingenuo", que se expresa en la captación fiel y exacta de los hechos materiales comprobables donde destacan epistemólogos y filósofos actuales de la talla de Russell, Moore y Bunge. Esta corriente filosófica se combina con la connotación gnoseológica (Fuente del Saber), sustentando el conocimiento a través del "empirismo" que sostiene que todo conocimiento se basa en la experiencia, donde destacan los filósofos Locke, Hume y J.S. Mill. En este sentido, la combinación de estas corrientes filosóficas acentuó las bases epistemológicas para el positivismo, una corriente que desde el siglo XIX promueve los métodos de las ciencias naturales a las sociales.

Augusto Comte (1798-1857) fue uno de los filósofos más destacados de esta 
corriente, al promulgar su obra Curso de Filosofía Positiva (1830-1842). Para Comte se destacan tres estados en la evolución del espíritu humano, donde se expresan el estado teológico, el estado metafísico y el estado positivo. Las posturas de Comte afirmaron que el último estado promulgado o estado positivo, llevaría al descubrimiento de leyes naturales y al dominio de la naturaleza a través del hombre.

Posteriormente, en 1920, surge el circulo de Viena, una corriente filosófica denominada positivismo lógico que se alejó de las posturas metafísicas, incorporando premisas cientificistas donde el significado de proposición es un modo de verificación a través de la evidencia empírica. En esta corriente destacan epistemólogos y filósofos de la talla de Schlick, Carnap, Neurath, Frank, Kauffman, Gödel, entre otros. Actualmente, el paradigma positivista se ha convertido en el dominante de la investigación social.

\subsubsection{Producción del modelo teórico}

La producción del modelo teórico en la investigación social está asociada intrínsecamente con el abordaje epistemológico (Empírico/Inductivo, Empírico/ Analítico o Empírico/Realista), que pretende verificar las hipótesis por medio de la observación o experimento, para ser replicables en cualquier tiempo y espacio como un hecho o predicción de los fenómenos sociales controlados por la evidencia empírica. En este sentido, el modelo teórico se concentra en la explicación de las relaciones causales entre variables verificables por la experiencia sensible para producir la teoría; esto a través de elementos conceptuales traducidos desde la inducción numérica o la inducción analítica. Según Bizquerra (2009), "los modelos que se utilizan en la investigación científica son configuraciones ideales que representan de manera simplificada una teoría" (p.33).

En atención a lo expuesto, la producción del modelo teórico se puede explicar por medio de la inducción numérica. Esto se realiza intentando traducir conceptualmente las certezas generales desde los hechos particulares del objeto de estudio, tomando en cuenta los resultados de la medición entre variables para predecir y explicar un fenómeno a través de un modelo representativo de la realidad, la cual actualmente es el más conocido adaptando un enfoque cuantitativo para medir los resultados de la investigación. De igual forma, el modelo teórico se puede explicar sistemáticamente a través de la inducción 
analítica, método desarrollado por Znaniecki (1882-1958), expuesto en su obra The Method of Sociology (1934), que consiste en verificar proposiciones teóricas sobre la naturaleza de la realidad social, donde se caracteriza la información recopilada a través de la evidencia empírica para generalizar por medio de la abstracción un modelo representativo de la realidad.

Ya en 1934, Znaniecki expresó, en The Method of Sociology, la fórmula de la inducción analítica como un procedimiento para verificar teorías y proposiciones. Aquí se reconoce su inscripción en la sociología clásica con enfoque naturalista (Augusto Comte, 1798-1857 y Emile Durkheim, 1858-1917), que se caracterizó por la admiración a los avances producidos en el seno de las ciencias naturales y formales. Pero se diferencia cuando pone el énfasis en comprender la perspectiva de los participantes de los hechos sociales. Esta es una característica innovadora que se inscribe en el enfoque cualitativo (Schettiny \& Cortazzo, 2015).

\subsection{Aproximación teórica en la investigación social}

La aproximación teórica en la investigación social se desarrolla a través del abordaje epistemológico (Racionalista/Deductivo, Racionalista/Crítico o Racionalista/Realista), circunscrito en el "paradigma postpositivista". En el abordaje de la investigación social se define el término "aproximación teórica", ya que concibe el conocimiento desde conjeturas o hipótesis que son aceptadas temporal o provisionalmente mientras resisten las pruebas de contrastación o falsación teórica; es decir; una teoría o hipótesis jamás podrá ser verificada, debido a que no existen verdades absolutas, pues siempre será posible su futura refutación con base en más datos, observaciones y experimentos. En este sentido, el investigador concibe una realidad relativa donde cualquier proposición debe someterse a revisión. Es por ello que todas las teorías se convierten en una aproximación.

La connotación ontológica (Ser) adopta una posición "realista", mejor conocida como "realismo crítico", que se expresa en someter a pruebas las teorías existentes a través del ensayo y error, conjetura o refutación, para falsearlas y proponer nuevas aproximaciones teóricas más cercanas a la realidad. Su máximo exponente es el filósofo Karl Popper (1902-1994), quién reemplaza la verificabilidad positivista por la falsabilidad postpositivista. Esta corriente 
filosófica se combina con la connotación gnoseológica (Fuente del Saber), sustentando el conocimiento a través del "racionalismo", donde el saber se obtiene a través de la razón mediante la deducción objetiva. Además de eso, Popper destaca el papel de la experiencia empírica en el marco de la refutación teórica y se le asigna el nombre de racionalismo crítico como una corriente filosófica moderna.

El enfoque epistemológico de Karl Popper se expone en su obra La lógica de la investigación científica (1935), Conjeturas y refutaciones (1963) y El conocimiento objetivo (1972). Sin embargo, en el Tercer Congreso Internacional de Lógica, Metodología y Filosofía de la Ciencia, Popper (1968) expone Epistemology Without a Knowing Subject, donde destaca una doctrina que manifiesta la interacción de los tres mundos del conocimiento. En el mundo uno, se conciben los objetos (el mundo universal); en el mundo dos, se conciben el mundo de los procesos mentales consientes e inconscientes (el subjetivo); y en el mundo tres, se concibe el conocimiento objetivo (los productos de la mente humana). Plantea así las bases del paradigma postpositivista.

\subsubsection{Producción de la aproximación teórica}

La producción de la aproximación teórica en la investigación social está asociada intrínsecamente con el abordaje epistemológico (Racionalista/Deductivo, Racionalista/Crítico o Racionalista/Realista), que pretende refutar las teorías científicas con conjeturas hipotético-deductivas. Esto debido a que considera que el conocimiento no puede ser concebido sobre la base de datos empíricos y pretender la generalización. En tal sentido, la aproximación teórica se sostiene sobre la base de la intuición del pensamiento para someter a prueba las teorías existentes, contrastarlas con la realidad y generar la falsación teórica desde un racionalismo crítico. De esta manera, se propone la aproximación teórica más cercana a la realidad, concibiendo que no hay leyes absolutas, sino imperfectas e inacabadas, donde todas las teorías deben ser refutadas o confirmadas.

Según Popper (1980), "las teorías científicas no son nunca enteramente justificables o verificables, pero que son, no obstante, contrastables. Diré, por tanto, que la objetividad de los enunciados científicos descansa en el hecho de que puedan contrastarse intersubjetivamente" (p.45). En atención a lo ex- 
puesto, Popper sostiene que la aproximación teórica emerge a través de conjeturas y refutaciones, donde se incorpora el valor de la experimentación para contrastar las teorías; la deducción lógica para hacer conjeturas; la intuición del pensamiento para formular hipótesis y enunciados; al igual que la intersubjetividad para dar validez, expresando el núcleo de la falsabilidad en su obra Conjeturas y Refutaciones (1963).

La transición de los datos a la teoría, en cambio, requiere de imaginación creadora. Popper (1963) observa que las teorías son el resultado de una intuición casi poética. Las hipótesis y teorías científicas no se derivan de los hechos observados, sino que se inventan para dar cuenta de ellos; son conjeturas relativas a las conexiones que se pueden establecer entre los fenómenos estudiados (Martínez Miguélez, 1998). Ciertamente, para la elaboración de una aproximación teórica, no existen recetas prestablecidas, ya que se traducen a través de conjeturas donde la intuición del pensamiento juega un rol estelar para encontrar los vacíos teóricos que se pretenden falsear. Sin embargo, se debe cumplir una estructura conceptual hipotético-deductiva, con las teorías sometidas a prueba, y demostrar su falsabilidad. Esto para proponer nuevas aproximaciones como resultado de esas conjeturas y refutaciones.

\subsection{Constructo teórico en la investigación social}

El constructo teórico en la investigación social se desarrolla a través del abordaje epistemológico (Introspectivo/Vivencial, Vivencialista/Experiencialista o Empírico/Idealista), circunscrito en el paradigma interpretativo. En el abordaje de la investigación social se define el término de "constructo teórico", ya que se concibe el conocimiento a través de una reconstrucción social que permite interpretar la realidad a través de la interacción entre los seres humanos, valorando las experiencias significativas de los contextos particulares mediados por las condiciones de tiempo y de espacio. En este sentido, el investigador concibe una realidad múltiple, donde convergen diversos factores que permiten interpretar la realidad, apreciando al ser en el mundo de un construccionismo y comprendiendo las experiencias vividas por medio del verstehen.

La connotación ontológica (Ser) adopta una posición idealista, mejor conocida como "idealismo trascendental". Esta concibe que el conocimiento humano está compuesto por juicios sintéticos a piori; es decir, sobre la base de 
una construcción intersubjetiva entre la sensibilidad y el entendimiento, que requiere una interacción cognoscitiva para formar el juicio de valor en la mente del sujeto. Su máximo exponente es el filósofo Immanuel Kant (17241804), quien expone su máxima obra, Critica de la Razón Pura (1781). Esta corriente, al combinarse con la gnoseología (Fuente del Saber), se soporta en el empirismo, para obtener el conocimiento desde lo sensible.

En oposición a las posturas filosóficas de Kant, surge la figura de Hegel (17701831), inclinando su posición filosófica hacia el idealismo objetivo. De esta manera, asume que la realidad puede ser percibida desde una conciencia individual, destacando el principio de la razón en una determinada sociedad. Posteriormente, la doctrina fenomenológica trasciende a través de Husserl (18591938). Este pensador combate los argumentos de la fenomenología del espíritu (1807) de Hegel, siendo crítico del psicologismo y proponiendo la fenomenología trascendental (1935), además de tomando en cuenta la concepción del ser del mundo desde una referencia cartesiana. En consecuencia, Heidegger (18891976), discípulo de Husserl, se distancia de su maestro y orienta su filosofía hacia el constructo del ser en la realidad, mediado por su temporalidad "ser en el mundo". Esto se reafirma con la promulgación de su obra Ser y Tiempo (1927), fundamentando al paradigma fenomenológico-hermenéutico.

\subsubsection{Producción del constructo teórico}

La producción del constructo teórico en la investigación social está asociada intrínsecamente con el abordaje epistemológico (Introspectivo/Vivencial, Vivencialista/Experiencialista o Empírico/Idealista), que pretende la comprensión del ser en el mundo, valorando los escenarios particulares de la realidad para reconstruir las experiencias sociales experimentadas por los sujetos significantes. En este contexto, el constructo teórico traduce el sentido de los datos extraídos de la realidad en un interaccionismo simbólico, tomando en cuenta la experiencia y agudeza intelectual del investigador para comprender los fenómenos a través de una teoría fundamentada en el construccionismo. Así, se sitúa al investigador como parte integral del proceso investigativo.

La teoría fundamentada construccionista se sostiene en la obra de Charmaz (2006), titulada Constructing Grounded Theory, que se expresa como una evolución de la teoría fundamentada clásica (grounded theory), desarrollada 
por B. Glaser y Strauss (1967). Esta propuesta pretendía crear teorías inductivas sobre la base objetiva de los datos empíricos, a través del rigor metodológico para cumplir con procedimientos analíticos.

La teoría fundamentada construccionista de Charmaz (2006) rescata el valor del sujeto investigador como un ente inextricable del proceso investigativo, que no se queda como un espectador recopilando datos en términos objetivos, sino que consigue altos niveles de abstracción analítica influenciado por su experiencia, reflexión y agudeza intelectual para la formación de conceptos generalizables que traduzcan la reconstrucción social a través del significado. Todo ello es posible en lo que se denomina "muestreo teórico", para la construcción emergente. La teoría fundamentada lleva a muestrear personas, entornos o estructuras más grandes, como agencias u organizaciones gubernamentales (Charmaz, 2006).

\subsection{Perspectiva teórica en la investigación social}

La perspectiva teórica en la investigación social se desarrolla a través del abordaje epistemológico (Racional/Subjetivista, Racional/Dialéctico o Racional/Idealista) circunscrito en la nueva versión del paradigma crítico. En el abordaje de la investigación social, se define el término "perspectiva teórica", debido a que el investigador está vinculado con su fenómeno en estudio inextricablemente y fija su posición investigativa desde una crítica social emancipadora mediada intersubjetivamente por los símbolos significantes del lenguaje en la acción comunicativa; esto con el objetivo de concebir una realidad que puede ser cuestionada en el mundo de la vida y los sistemas sociales. En consecuencia, el investigador procura la emancipación a través de la reflexión crítica del saber y la racionalidad expresada en la acción comunicativa.

La connotación ontológica (Ser) adopta una posición "idealista", mejor conocida como "idealismo objetivo", donde el conocimiento se concibe como un concepto único fundamentado en la razón, ya que está vinculado a un orden histórico definido con un trasfondo político. Su máximo exponente es Georg Hegel (1770-1831), quien fundamentó su pensamiento filosófico en las obras Fenomenología del Espíritu (1807), La Ciencia de la Lógica (1812) y Filosofía de la Historia (1837), convirtiéndose en un crítico del idealismo trascendental propuesto por Kant (1724-1804). 
Hegel concibe la naturaleza humana en términos históricos, impulsada por un proceso de contrastación tesis-antítesis-y-síntesis, denominado proceso dialéctico. Esta corriente, al combinarse con la gnoseología (Fuente del Saber), expresa un fundamento del saber desde el racionalismo, como vía del conocimiento basado en la intuición. Desde la basa hegeliana partió un conjunto heterogéneo de corrientes que imprimieron diversidades, resumidas entre el estructuralismo, posestructuralismo y postmodernismo, destacando la relevancia de la Escuela de Frankfurt (Alemania), con filósofos de la talla de Horkheimer, Adorno, Marcuse y Fromm. En la segunda generación de Frankfurt, surgen filósofos como Habermas, que en su obra La acción Comunicativa (1981) supera contradicciones filosóficas del pasado entre materialistas y trascendentales. Habermas asume el idealismo objetivista, mediado por la acción comunicativa del lenguaje, en una relación intersubjetiva del saber, asignando una actualización del paradigma crítico.

\subsubsection{Producción de la perspectiva teórica}

La producción de la perspectiva teórica en la investigación social está asociada intrínsecamente con el abordaje epistemológico (Racional/Subjetivista, Racional/Dialéctico o Racional/Idealista). Se pretende cuestionar e interpretar la realidad social en el "mundo de la vida", tomando en cuenta la cultura que rige la sociedad, influenciando el comportamiento de los individuos en un "sistema" (político, económico o social) que requiere su transformación a partir de la reflexión crítica del investigador para proponer elementos conceptuales desde la acción comunicativa del lenguaje. Estos fundamentos, para generar la perspectiva teórica, se traducen desde la teoría crítica, la hermenéutica, la filosofía analítica del lenguaje, la teoría de sistemas y la antropología de la cultura circunscrita en la acción comunicativa de Habermas (1981).

Habermas fue considerado un neomarxista. Sus nuevos trabajos lo alejan de tal posición e incluso de la teoría crítica. Sus planteamientos teóricos más actuales lo han llevado a interesarse por las ideas de Mead, Parsons, Schutz y Durkheim, a pesar de que sus preocupaciones principales siguen siendo la acción comunicativa y el proceso de racionalización según la versión weberiana (Briones, 2002). En atención a lo expuesto, la perspectiva teórica debe producirse desde una constitución intersubjetiva, a través de una teoría que fundamente el análisis de las situaciones complejas circunscritas en el 
sistema social en que se desenvuelve el objeto de estudio. En consecuencia, resulta pertinente proponer la producción de la perspectiva teórica tomando en cuenta la obra de Clarke (2005), titulada Situational analysis: grounded theory after postmodern turn. En ella se critica el muestreo teórico de la teoría fundamentada del construccionismo, para proponer las bases de la teoría fundamentada postmodernista, mejorando sus postulados en la segunda edición de su obra: Situational analysis Grounded theory after the interpretive turn (2017).

En consecuencia, la teoría fundamentada, después del giro interpretativo, expone el análisis situacional a través de diversos recursos (entrevistas, observaciones, material de discurso histórico, narrativo y visual), para comprender y cuestionar las situaciones del mundo de la vida. En este sentido, la teoría se propone a partir del análisis situacional, incorporando tres mundos que Clarke define como mapas situacionales, sociales y posicionales.

\subsection{Evaluación de teorías en la investigación social}

La producción de teorías expuestas en las secciones anteriores está lejos de pretender constituirse como una receta instrumentalista para proponer un modelo, aproximación, constructo o perspectiva teórica en la investigación social. Esto debido a que la teoría obedece a una invención creativa del investigador, quien está influenciado por su agudeza intelectual para estructurar esquemas conceptuales que expongan la intencionalidad epistemológica como vía para acceder al conocimiento científico. En este escenario, algunos criterios expuestos son tomados en cuenta en la evaluación teórica. Desde esta perspectiva, en este estudio se sostiene que la legitimización de la investigación y, por ende, de la teoría que se construyó como consecuencia de esta, podría hacerse desde su propia estructura interna, asociada al sistema de conceptos, instrumentos, palabras, que usa el investigador para nombrar, describir o explicar el objeto de estudio o el fenómeno social abordado en la investigación (Piñero \& Rivera, 2021).

Se tienen cuatro formas de conocimiento teórico derivadas de la epistemología, de la perspectiva de investigación, del problema de la investigación y de los métodos que se pretende utilizar. Todas estas formas de conocimiento desempeñan un rol implícito o (mejor) explicito en el modo de hacer investi- 
gación y, antes de eso, en el modo en que esta se plantea (Flick, 2007). En concordancia con lo expuesto, y tomando en cuenta la enriquecedora experiencia de consultar diversos autores que orientaron la fundamentación de la investigación, se proponen algunos criterios referenciales para la evaluación de modelos, aproximaciones, constructos y perspectivas teóricas, exponiendo los siguientes:

\section{Figura 1}

\section{Criterios para la evaluación de teorías}

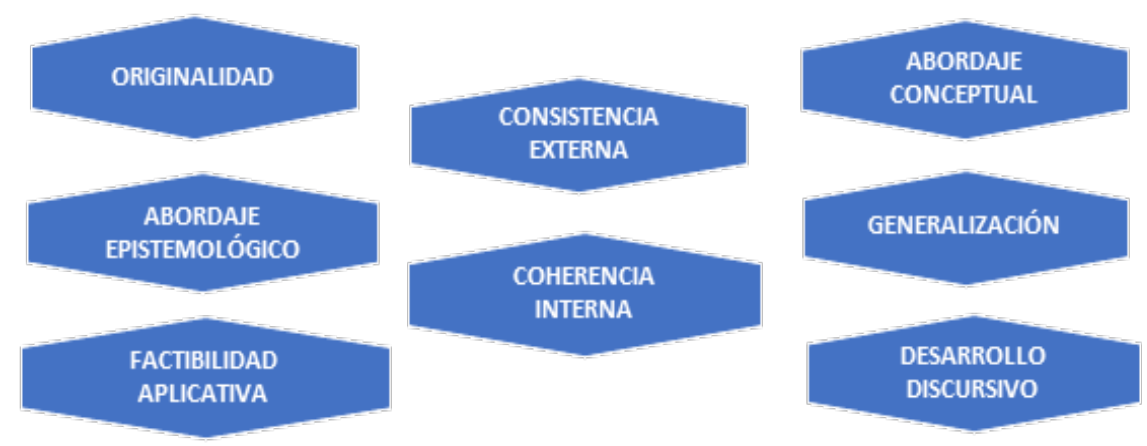

\section{Nota: Elaboración propia.}

\section{Consideraciones finales}

Finalmente, la investigación considera que la orientación para la producción de teorías científicas se sostiene sobre la epistemología y sus diferentes formas de abordaje para acceder al conocimiento, a través de un eje perceptivo, relacional y procedimental que debe registrar la acción discursiva del lenguaje inherente a la intencionalidad del investigador, tomando en cuenta el enfoque investigativo. Se hace hincapié en las siguientes consideraciones:

- La percepción del investigador frente a la realidad social es inherente a la connotación ontológica del ser, expresada entre el "idealismo y el realismo", y asumiendo que el idealismo es una corriente filosófica con diversos matices que se traduce en la posición del ser como resultado de su pensar para percibir la realidad tomando en cuenta un carácter subjetivo del investigador; mientras que el realismo es otra corriente filosófica con diversos matices que condiciona el pensar previo a la posición del ser para percibir la realidad objetivamente. En tal sentido, el investigador, en 
su abordaje, pudiera adoptar dos perspectivas incompatibles de la realidad: Idealismo/Subjetivista vs Realismo/Objetivista.

- La relación entre el investigador y lo investigado para acceder al conocimiento científico es inherente a la postura epistemológica adoptada en el proceso investigativo. Esto debido a que se manifiestan diversas formas de abordaje para conocer la realidad en estudio. En consecuencia, se origina una relación entre la posición del investigador y la fuente del saber (Ser/Saber; Ontología/gnoseología), produciendo una relación Investigador/Investigado en cuatro formas predominantes: Empirico/Inductivo, Empírico/Analítico o Empírico/Realista; Racionalista/Deductivo, Racionalista/Crítico o Racionalista/Realista; Introspectivo/Vivencial, Vivencialista/Experiencialista o Empírico/Idealista; y Racional/Subjetivista, Racional/Dialéctico o Racional/Idealista.

- La metodología de la investigación responde los objetivos del estudio, según la realidad social abordada y la fuente del conocimiento al que se pretende acceder. Es decir, a la intencionalidad del investigador para verificar hipótesis a través de la evidencia empírica, refutar teorías a través del falsacionismo, comprender la realidad social mediante la reconstrucción de las experiencias vivenciales o cuestionar la estructura del sistema para su transformación a través de la acción comunicativa.

\section{Fuentes consultadas}

Bauer, M., \& Gaskell, G. (2008). Pesquisa Qualitativa com Texto: Imagem e Som: Um Manual Prático. Petrópolis. https://tecnologiamidiaeinteracao.files.wordpress.com/2017/10/pesquisa-qualitativa-com-texto-imagem-e-som-bauer-gaskell.pdf

Bizquerra, R. (2009). Metodología de la Investigación Educativa. Editorial La Muralla.

Briones, G. (2002). Epistemología de las Ciencias Sociales. ICFES.

Camacho, H., Fontaines, T., \& Urdaneta, G. (2005). La trama de la investigación y su epistemología. Revista Telos, 7(1), 09-20. https://www. redalyc.org/pdf/993/99318830001.pdf 
Cárcamo, H., Méndez, P., \& Rebolledo, A. (2008). (10-12 de diciembre de 2008). Expresión del debate cuantitativo/cualitativo en las ciencias de la educación. I Encuentro Latinoamericano de Metodología de las Ciencias Sociales, La Plata, Argentina. http://www. memoria.fahce.unlp.edu.ar/trab_eventos/ev.9463/ev.9463.pdf

Carnap, R. (1965). Conocimiento y verdad. En A. Ayer (Ed.), El Positivismo Lógico (pp.169-204). Fondo de Cultura Económica. https:// lenguajeyconocimiento.files.wordpress.com/2015/04/el-positivismo-logico.pdf

Charmaz, K. (2006). Constructing Grounded Theory. Sage Publications. http://www.sxf.uevora.pt/wp-content/uploads/2013/03/Charmaz_2006.pdf

Chaves, A., \& Gadea, W. (2018). La Relación Subjeto-Objeto en la concepción kantiana de la ciencia. Sophia, 1(25). https://www.redalyc.org/ jatsRepo/4418/441855948003/html/index.html

Clarke, A. (2005). Situational analysis: grounded theory after postmodern turn. Sage Publications.

Clarke, A., Friese, C., \& Washburn, R. (2017). Situational analysis Grounded theory after the interpretive turn. Sage Publications.

Comte, A. (1981). Curso de Filosofía Positiva. Primera y Segunda lecciones. Aguilar.

Corbetta, P. (2007). Metodología y Técnicas de Investigación Social. McGraw Hill. https://luisdoubrontgschool.files.wordpress.com/2021/04/ corbetta-metodologia-y-tecnicas-de-investigacion-social.pdf

Crotty, M. (1998). The Foundations of Social Research. Sage Publications. https://seminariodemetodologiadelainvestigacion.files. wordpress.com/2012/03/the-foundations-of-social-researchmeaning-and-perspective-in-the-research-process-michael-crotty.pdf

De Berrios, O., \& Briceño, M. (2009). Enfoques epistemológicos que orientan la investigación de 4to. nivel. Revista Visión Gerencial, 1(8), 47-54. https://www.redalyc.org/pdf/4655/465545882009.pdf 
Doubront, M., \& Doubront, L. (2020). Impacto del contexto económico, social y político de Venezuela en el docente universitario. Análisis desde la Pirámide de Maslow. Dissertare, 5, 1-15. https://revistas.uclave. org/index.php/dissertare/article/view/2791/1749

Flick, U. (2015). El Diseño de la Investigación Cualitativa. Morata.

Foucault, M. (1970). La Arqueología del Saber. Editions Gallimard. https://luisdoubrontgschool.files.wordpress.com/2021/04/foucault-michel-la-arqueologia-del-saber.pdf

Galeano, M. (2012). Estrategias de Investigación Social Cualitativa. La Carreta Editores

Glaser, B., \& Strauss, A. (1967). Basics of qualitative research. Techniques and procedures for developing grounded theory. Sage Publications.

Guba, E., \& Lincoln, Y. (1998). Competing Paradigms Qualitative Research. En N. Denzin, \& Y. Lincoln (eds.), The Landscape Qualitative Research. Sage.

Habermas, J. (1999). Teoría de la acción comunicativa. Taurus.

Hegel, G. (1807). System der Wissenschaft. Erster Teil, die Phänomenologie des Geistes. Bamberg und Würzburg.

Hegel, G. (2011). Ciencia de la Lógica. Abada Editores.

Hegel, G. (2016). Filosofía de la Historia. Ediciones Losada.

Heidegger, M. (1996). Ser y Tiempo. Trotta.

Hessen, J. (1999). Teoría del Conocimiento. ILCA.

Hincapié, J. (2017). Elementos ontológicos, epistemológicos y metodológicos para la construcción de un marco teórico de estudio de los intangibles. Revista Cuadernos de Contabilidad, 18(45), 86-109. https:// doi.org/10.11144/Javeriana.cc18-45.eoem

Horkheimer, M. (2000). Teoría Tradicional y Teoría Crítica. Paidós.

Husserl, E. (1984). Crisis de las ciencias europeas y la fenomenología trascendental. Folios. 
Husserl, E. (1985). Meditaciones Cartesianas. Fondo de Cultura Económica.

Husserl, E. (1993). Ideas Relativas a una Fenomenología Pura y una Filosofía Fenomenológica. Fondo de Cultura Económica.

Kant, I. (2000). Crítica de la Razón Pura. Alfaguara.

Martínez, M. (1998). La Investigación Cualitativa Etnográfica en Educación. Trillas.

Meléndez, L., \& Pérez, C. (2006). Propuesta Estructural para la construcción metodológica en investigación cualitativa como dinámica del conocimiento social. Revista Venezolana de Información, Tecnología y Conocimiento Enlace, 3(3), 33-50. http://ve.scielo.org/scielo. php?script=sci_arttext\&pid=S1690-75152006000300003

Nava, J. (2017). La esencia del conocimiento. El problema de la relación sujeto-objeto y sus implicaciones en la teoría educativa. Revista Iberoamericana para la Investigación y el Desarrollo Económico-RIDE, 8(15), 25-57. https://doi.org/10.23913/ride.v8i15.289

Ortiz, E. (2013). Epistemología de la investigación cuantitativa y cualitativa: paradigmas y objetivos. Revista de Clases Historia, 2(408), 2-23. https://www.researchgate.net/publication/303313448_Epistemologia_de_la_InvestigacionCuantitativa_y_Cualitativa_Paradigmas_y_Objetivos

Padrón, J. (2014). Proyecto de Epistemología en DVD. Doctorado en Ciencias Humanas. La Universidad del Zulia. http://padron.entretemas. com.ve/Notas_EP-EnfEpistPdigmas.pdf

Paz-Sandín, E. (2003). Investigación cualitativa en educación. Fundamentos y tradiciones. McGraw Hill. https://uisdoubrontgschool.files. wordpress.com/2021/03/paz-sandin-esteban_investigacion-cualitativa-en-educacion_-.pdf

Perdomo, E. (2009). Michael Foucault. La Arqueología del Saber. Revista Textuales, 1(3), 135-141. http://www.saber.ula.ve/bitstream/handle/123456789/28600/articulo9_3.pdf?sequence=1\&isAllowed=y

Piñero, M., \& Rivera, M. (2013). Investigación Cualitativa. Orientaciones Procedimentales. Fedupel. 
Popper, K. (1935). Logik der Forschung. Julius Springer Verlag.

Popper, K. (1963). Conjectures and Refutations: The Growth of Scientific Knowledge. Routledge.

Popper, K. (1968). Epistemology Without a Knowing Subject. ELSEVIER

ScienceDirect, 52, 333-373. https://doi.org/10.1016/S0049-237X(08)71204-7

Popper, K. (1972). Objective Knowledge: An Evolutionary Approach. Clarendon Press.

Popper, K. (1980). La Lógica de la Investigación Científica. Tecnos. http:// www.raularagon.com.ar/biblioteca/libros/Popper\%20Karl\%20-\%20 La\%20Logica\%20de\%201a\%20Investigacion\%20Cientifica.pdf

Ramírez, F., \& Zwerg, A. (2012). Metodología de la investigación: más que una receta.

AD Minister, 12(20), 91-111. https://core.ac.uk/download/pdf/290651523. pdf

Sautu, R. (2005). Todo es Teoría. Objetivos y Métodos de Investigación. Lumiere. https://luisdoubrontgschool.files.wordpress.com/2021/04/ruth-sautu-odo_es_teoria_objetivos_y_metodos_en_investigacion sautu_ruth.pdf

Schettiny, P., \& Cortazzo, I. (2015). Análisis de Datos Cualitativos en la Investigación

Social. Procedimientos y herramientas para la interpretación de la información cualitativa. Editorial de la Universidad de La Plata.

Severini, L. (1961). Existencialismo. Herder.

Vidal, J. (2013). La búsqueda de la realidad o de la verdad: una aproximación a partir de la teoría sociológica. Cinta de Moebio, (47), 95-114. http://dx.doi.org/10.4067/S0717-554X2013000200004

Yánez, P. (2018). Estilos de pensamiento, enfoques epistemológicos y la generación del conocimiento científico. Revista Espacios, 39(51). https://www.revistaespacios.com/a18v39n51/a18v39n51p18.pdf

Znaniecki, F. (1934). The Method of Sociology. Farrar \& Rinehart. 